\title{
Differential deposition to correct surface figure deviations in astronomical grazing-incidence $X$-ray optics
}

\author{
Kiranmayee Kilaru, Brian D. Ramsey ${ }^{2}$, Mikhail V. Gubarev ${ }^{2}$ \\ ${ }^{1}$ NASA Postdoc Program Associate \\ ${ }^{2}$ NASA Marshall Space Flight Center (MSFC), Huntsville, AL, 35812
}

\begin{abstract}
A coating technique is being developed to correct the surface figure deviations in reflectivegrazing-incidence X-ray optics. These optics are typically designed to have precise conic profiles, and any deviation in this profile, as a result of fabrication, results in a degradation of the imaging performance. To correct the mirror profiles, physical vapor deposition has been utilized to selectively deposit a filler material inside the mirror shell. The technique, termed differential deposition, has been implemented as a proof of concept on miniature X-ray optics developed at MSFC for medical-imaging applications. The technique is now being transferred to larger grazing-incidence optics suitable for astronomy and progress to date is reported.
\end{abstract}

Keywords: Grazing incidence X-ray optics, Differential deposition, Surface figure deviations in X-ray optics 